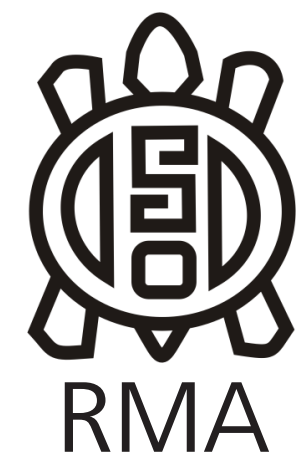

Dossier

\title{
La obsidiana en la ocupación tardía de Cueva Nacimiento I, Puna de Salta, Argentina (ca. 500 años A.P.)
}

\author{
Obsidian in the late context of Cueva Nacimiento I, Puna of \\ Salta, Argentina (ca. 500 years B.P.) \\ María Varde*
}

*María Varde, CONICET, Instituto de Arqueología, Facultad de Filosofía y Letras, Universidad de Buenos Aires, Argentina. E-mail: mar_vd@yahoo.com

\begin{abstract}
Resumen
En este trabajo se presenta el análisis del uso de la obsidiana en el conjunto lítico de Cueva Nacimiento 1, en un contexto agropastoril tardío. Los resultados indican que la obsidiana fue un recurso utilizado de una manera que maximizó su aprovechamiento dentro de una estrategia tecnológica mixta de conservación-expeditividad. La comparación con las materias primas locales permite afirmar que a pesar de la disponibilidad local de rocas de buena calidad, la frecuencia de obsidiana en el conjunto es predominante, y su uso se orientó a la confección de instrumentos formatizados de alta y baja inversión de energía. Estos resultados plantean la posibilidad de que los grupos que utilizaron la cueva en momentos tardíos hayan formado parte de redes de interacción de amplio alcance de manera persistente. Este acceso relativamente abierto a materiales de fuentes diversas pudo ofrecer la posibilidad de una estrategia tecnológica que combinó artefactos de carácter conservado y otros expeditivos sobre materias primas de excelente calidad.
\end{abstract}

Palabras clave: Obsidiana; Estrategias tecnológicas; Redes de interacción; Período Tardío; Puna argentina.

\begin{abstract}
We present the results of the study of the use of obsidian in the lithic set of Cueva Nacimiento 1 in a late agropastoral context. The results indicate that obsidian was a resource used in a way that maximized it within a mixed technological strategy of conservation-expediency. At the same time, the comparison with the local raw materials allows to affirm that in spite of the local availability of good quality rocks, the frequency of obsidian in the set is predominant, and its use is destined to the manufacture of instruments of high and low energy investment. These results raise the possibility that the groups that used the cave in Late period moments could have been part of persistent interaction networks of wide scope. This relatively open access to materials from different sources could offer the possibility of a technological strategy that combined artifacts of conserved nature and other expeditious ones on raw materials of excellent quality.
\end{abstract}

Key words: Obsidian; Technologies strategies; Interaction networks; Late period; Puna of Argentina.

\section{Introducción}

Los estudios de la tecnología lítica de ocupaciones agropastoriles del Período Tardío e Inka de la Puna argentina (ca. 1000-500 AP) documentaron una fuerte tendencia hacia estrategias expeditivas en la reducción de núcleos y en la formatización de los instrumentos, con altas frecuencias de materias primas locales e inmediatas (Elías, 2010; Escola, Elias y Paulides, 2006). Esto se relacionó con la intensificación del sedentarismo y con los riesgos productivos propios de las economías agropastoriles (Escola, 2004; Elias, 2010). En este contexto, la presencia de materias primas no locales, específicamente obsidiana, se halló generalmente asociada a estrategias conservadas, en la manufactura de puntas de proyectil (Elías, 2010; Escola et al., 2006; Lavallé et al., 1997). Este hecho fue relacionado con la búsqueda de artefactos confiables orientados a cumplir con eficacia determinadas funciones (caza, defensa) (Escola et al., 2006; Elias, 2010), así como también se ha argumentado que el uso privilegiado de obsidiana para las puntas de proyectil pudo tener una raíz de naturaleza social (Carbonelli, 2014). En última instancia, la presencia de rocas alóctonas en estos contextos fue vinculada con la intensificación creciente de las redes de interacción que se registró en la región desde el periodo Temprano o Formativo (Elias y Escola, 2010; Escola, 2004a; Mercuri, 2011; Yacobaccio, Escola, Pereyra, Lazzari y Glascock, 2004). A partir del análisis 
de la frecuencia y los patrones de uso de las distintas variedades de obsidiana por parte de las poblaciones agropastoriles se evaluó su participación dentro de la dinámica regional de interacción e intercambio (Escola y Elias, 2010; Escola, 2004a; Escola, Elias y Cohen, 2016; Escola, Glascock, Korstanje y Sentinelli, 2009; Mercuri, 2011; Yacobaccio et al, 2004).

En este marco, una forma de analizar la diversidad de las estrategias es la estimación de la inversión de energía en la manufactura de los instrumentos líticos (Escola, 2004b; Hocsman y Escola, 2006-2007). Esta línea de trabajo propone observar el tratamiento tecnológico diferencial mediante la evaluación de la clase técnica, es decir, el esfuerzo de formatización de los instrumentos, de acuerdo con la secuencia, superposición y extensión de los lascados respecto de las caras y los bordes de la pieza (Aschero y Hocsman, 2004) en relación con la noción de diseño (Escola, 2004b). De esta manera es posible observar diferencias y similitudes en el aprovechamiento de materias primas locales y no locales para la confección de instrumentos formatizados. En última instancia, estos aspectos de la organización tecnológica pueden ofrecer información del rol que tuvieron los materiales no locales en las estrategias desplegadas dentro de la dinámica económica general de una ocupación.

En la puna de Salta, estos análisis se han enfocado en conjuntos correspondientes a ocupaciones tempranas de las cuencas de San Antonio de los Cobres y Santa Rosa de los Pastos Grandes (Mercuri, 2011), pero aún no se han realizado en conjuntos provenientes de contextos más tardíos. Para contribuir con esta línea de estudio, en este trabajo se presenta el análisis del uso de obsidiana no local en un contexto tardío de la Puna de Salta, en términos de inversión de energía, de manera de caracterizar las estrategias tecnológicas involucradas (Hocsman y Escola, 2006/2007). En última instancia, se espera relacionar los resultados con el contexto más general de los antecedentes regionales.

\section{Antecedentes: Cueva Nacimiento 1}

El sitio Cueva Nacimiento 1, a $4200 \mathrm{msnm}$ y $18 \mathrm{~km}$ al sur de San Antonio de los Cobres, Provincia de Salta, Argentina (Figura 1), forma parte de un sistema de tres cuevas utilizadas actualmente por los pastores para encierro de animales y actividades domésticas. La ocupación tardía del sitio tiene un fechado radiocarbónico de $495 \pm 30$ años AP. La estratigrafía de esta ocupación presenta lentes de guano intercaladas con lentes carbonosas asociadas a estructuras de combustión. En estas últimas se halló una alta frecuencia de artefactos líticos, restos arqueofaunísticos, restos arqueobotánicos, camadas de paja, un marlo, y tiestos cerámicos similares a los de Tastil. De los restos faunísticos identificables $(\mathrm{N}=332)$, se determinó que los camélidos superan el 99\%, y los tres especímenes restantes se asignaron a vizcacha (Lagidium Viscacia) (Varde et al., 2017). Los restos de camélidos muestran una fuerte incidencia de termoalteración detectada en un $70 \%$, y un $18 \%$ muestra marcas antrópicas de diferente clase. Los resultados del análisis osteométrico realizado sobre 16 especímenes muestran dos rangos de tamaño, uno correspondiente a llama $(12,5 \%)$ y el segundo a vicuña $(87,5 \%)$ (Varde et al., 2017). La tecnología lítica registró una gran abundancia de desechos de talla, 8 núcleos no formales y 80 instrumentos relacionados principalmente con la caza y el procesamiento de recursos faunísticos (Varde et al., 2017). Las puntas de proyectil dominan el conjunto de los instrumentos formatizados. Estos artefactos tienen en su gran mayoría una morfología triangular apedunculada, y son de tamaño pequeño y espesor muy delgado, con sólo dos puntas mediano pequeñas de espesor delgado (Figura 2).

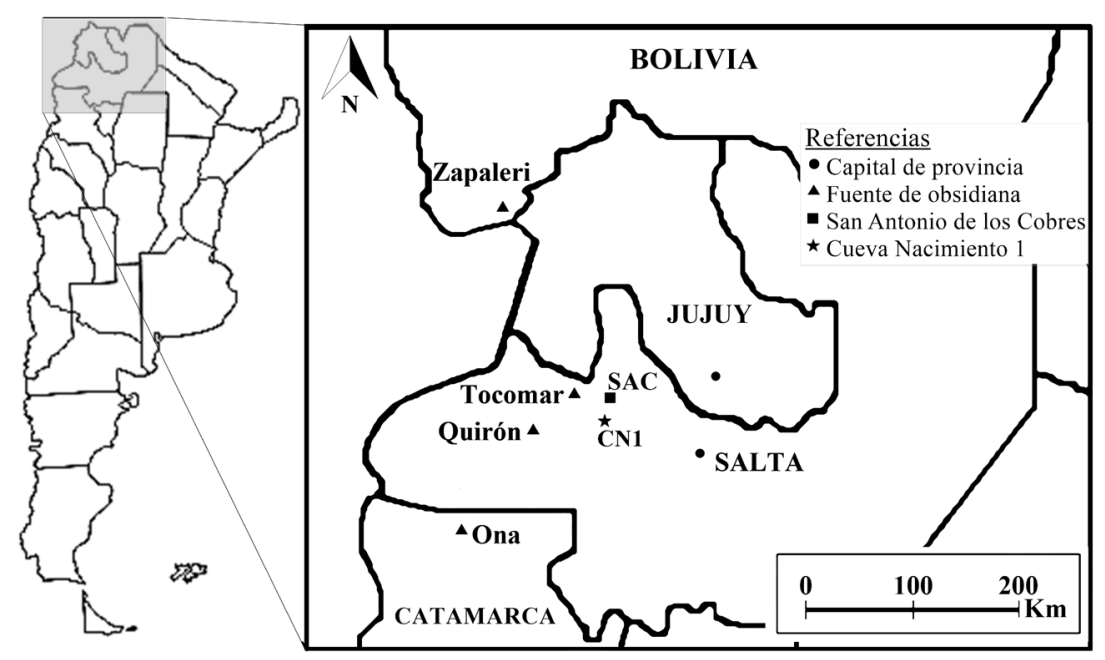

Figura 1. Localización geográfica de Cueva Nacimiento 1 y las fuentes de obsidiana mencionadas en el texto.

Figure 1. Geographic location of Cueva Nacimiento 1 and sources of obsidian mentioned in the text. 
La materia prima predominante tanto en los desechos de talla como en los instrumentos es la obsidiana, cuyas fuentes fueron identificadas mediante un análisis de Fluorescencia de Rayos X de Energía Dispersiva (EDXRF) de seis artefactos representativos de las distintas variedades de obsidiana discriminadas previamente a ojo desnudo, a cargo del Dr. Michael Glascock en el Missouri University Research Reactor (MURR). Los resultados indicaron que las variedades de obsidiana de los artefactos analizados corresponden a las fuentes de Zapaleri, Tocomar, Quirón, y Ona. Estos resultados coinciden con la clasificación realizada a ojo desnudo, y confirman que todas las variedades de obsidiana presentes en la muestra provienen de fuentes no locales (Figura 1), tomando como no local toda fuente que supera una distancia de $25 \mathrm{~km}$ respecto del sitio (López, 2008). Macroscópicamente, la variedad de Quirón se muestra brillante, traslúcida e incolora con pequeñas inclusiones negras. Tocomar en cambio es opaca traslúcida grisácea con inclusiones aisladas, y pude presentar vetas o bandas de color más claro o blancas. La variedad Zapaleri es brillante negra, y la variedad Ona se presenta brillante traslúcida con o sin inclusiones aisladas y vetas o bandas grises bien definidas, que en muchos casos forman rectas paralelas. El resto de las materias primas del conjunto son de origen local, y en general son de buena calidad para la talla. Estas consisten en sílices de grano fino de distintos colores, toba silicificada roja, cuarzo, ópalo, cuarcita, andesita afanítica (Varde et al, 2017).

Sobre la base de este registro arqueológico se propuso que la cueva fue utilizada alternadamente como lugar de encierro de llamas y como un espacio de habitación humana en tiempos prehispánicos, donde la caza fue una actividad importante, a juzgar por los datos osteométricos y la abundante presencia de puntas de proyectil (Varde et al. 2017).

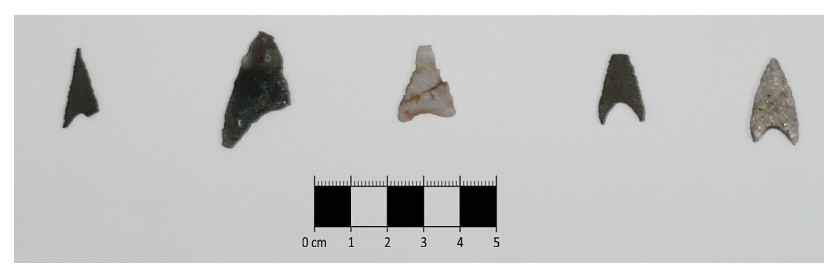

Figura 2. Algunas puntas de proyectil de la ocupación tardía de Cueva Nacimiento 1.

Figure 2. Some projectile points of the late component of Cueva Nacimiento 1.

\section{Objetivos, muestra y metodología}

En este trabajo se busca documentar los patrones de uso de las materias primas líticas no locales en la ocupación tardía de Cueva Nacimiento 1 para obtener información acerca del rol que cumplieron estos materiales en las estrategias tecnológicas implementadas. Para esto, se realizó un análisis tecnomorfológico (sensu Aschero 1983) de todos los instrumentos líticos realizados por lascado $(\mathrm{N}=72)$, se los clasificó según grupo tipológico y se los caracterizó en términos de inversión de trabajo por clase técnica (Hocsman y Escola, 2006/2007). Así, en orden decreciente de trabajo invertido en la formatización, se clasifican el adelgazamiento bifacial, la reducción bifacial, el adelgazamiento unifacial, la reducción unifacial, el trabajo no invasivo bifacial, el trabajo no invasivo unifacial, el trabajo no invasivo alternante y el trabajo bipolar (Aschero y Hocsman, 2004). También se observó los requerimientos de extracción de la forma base, de formatización de la forma base y de imposición de forma del diseño, de acuerdo con Hocsman y Escola (2006/2007). Los caracteres que se tuvieron en cuenta son ancho de los lascados sobre el borde, extensión de los lascados sobre las caras, continuidad de los lascados

Tabla 1. Grupos tipológicos del conjunto según clase de materia prima.

Table 1. Typology groups by type of raw material

\begin{tabular}{|c|c|c|c|c|c|c|c|c|c|c|c|}
\hline \multirow{2}{*}{ GRUPO TIPOLOGICO } & \multicolumn{9}{|c|}{ Obsidiana } & \multicolumn{7}{|c|}{ Locales } \\
\cline { 2 - 16 } & Quirón & Tocomar & Ona & Zapaleri & Silice & Andesita & Toba & Cuarzo & Cuarcita & Ópalo & Total \\
\hline Puntas de proyectil y preformas & 10 & 3 & 5 & 3 & 8 & 5 & 1 & 1 & & & 36 \\
\hline Cuchillo & 1 & & 2 & & & 2 & & & & & 5 \\
\hline Filo en punta & & 1 & & & 2 & 1 & & & & & 4 \\
\hline Fragmento no diferenciado & 4 & 2 & & 1 & 2 & & & & & & 9 \\
\hline Raspador & 2 & & 1 & & 1 & & & & & & 4 \\
\hline Retoque sumario & 1 & & 3 & 1 & 2 & 1 & 2 & 2 & 1 & 1 & 14 \\
\hline Muesca retocada & & & & & & & & 1 & & & 1 \\
\hline perforador & & & & 1 & & & & & & & 1 \\
\hline Filo formatizado no diferenciado & & 2 & & & & & & 1 & & & 3 \\
\hline \hline TOTAL & 18 & 8 & 11 & 6 & 15 & 9 & 3 & 5 & 1 & 1 & 77 \\
\hline
\end{tabular}


Tabla 2. Detalle de las clases técnicas de los instrumentos por materia prima.

Table 2. Detail of the technical class of the instruments by type of raw material.

\begin{tabular}{|c|c|c|c|c|c|c|c|c|c|c|c|}
\hline & \multicolumn{9}{|c|}{ CLASE TÉCNICA } \\
\hline & & & $\begin{array}{c}\text { adelg. } \\
\text { bifacial }\end{array}$ & $\begin{array}{l}\text { reducción } \\
\text { bifacial }\end{array}$ & $\begin{array}{c}\text { adelg. } \\
\text { unifacial }\end{array}$ & $\begin{array}{c}\text { reducción } \\
\text { unifacial }\end{array}$ & $\begin{array}{c}\text { trabajo no } \\
\text { invasivo } \\
\text { bifacial }\end{array}$ & $\begin{array}{c}\text { trabajo no } \\
\text { invasivo } \\
\text { unifacial }\end{array}$ & $\begin{array}{l}\text { trabajo no } \\
\text { invasivo } \\
\text { alternante }\end{array}$ & $\begin{array}{l}\text { trabajo } \\
\text { bipolar }\end{array}$ & TOTAL \\
\hline \multirow{12}{*}{$\begin{array}{l}\text { MATERIA } \\
\text { PRIMA }\end{array}$} & \multirow{7}{*}{ LOCAL } & Silices & 0 & 8 & 0 & 0 & 2 & 4 & 0 & 0 & 14 \\
\hline & & Andesita & 0 & 6 & 0 & 0 & 2 & 1 & 0 & 0 & 9 \\
\hline & & Cuarzo & 0 & 1 & 0 & 0 & 1 & 2 & 0 & 0 & 4 \\
\hline & & Toba & 0 & 1 & 0 & 0 & 0 & 2 & 0 & 0 & 3 \\
\hline & & Cuarcita & 0 & 0 & 0 & 0 & 0 & 1 & 0 & 0 & 1 \\
\hline & & Ópalo & 0 & 0 & 0 & 0 & 0 & 1 & 0 & 0 & 1 \\
\hline & & SUBTOTAL & 0 & 16 & 0 & 0 & 5 & 11 & 0 & 0 & 32 \\
\hline & \multirow{5}{*}{ OBSIDIANA } & Quirón & 0 & 11 & 0 & 1 & 1 & 3 & 0 & 0 & 16 \\
\hline & & Tocomar & 0 & 5 & 0 & 0 & 3 & 0 & 0 & 0 & 8 \\
\hline & & Ona & 0 & 5 & 0 & 0 & 0 & 4 & 1 & 0 & 10 \\
\hline & & Zapaleri & 0 & 3 & 0 & 1 & 1 & 0 & 1 & 0 & 6 \\
\hline & & SUBTOTAL & 0 & 24 & 0 & 2 & 5 & 7 & 2 & 0 & 40 \\
\hline \multicolumn{2}{|c|}{ TOTAL } & & 0 & 40 & 0 & 2 & 10 & 18 & 2 & 0 & 72 \\
\hline
\end{tabular}

sobre el borde, situación de los lascados con respecto a las caras de la pieza, forma primaria del filo, forma del filo sobre la arista, extensión del filo en relación a los bordes de la pieza, y forma base (Aschero, 1983). También se observó la presencia de reactivación en los filos activos, como indicador de la intención de prolongar la vida activa de los artefactos.

\section{Resultados}

La Tabla 1 muestra la clasificación por grupo tipológico de los instrumentos de obsidiana y los de materias primas locales. Mientras que ambas categorías se hallan representadas en ocho de los nueve grupos tipológicos, las rocas locales dominan en sólo tres de esos grupos. Con respecto a las variedades de obsidiana, se observa que en general todas se hallan representadas en una cantidad similar de grupos tipológicos, siendo Quirón la más abundante, seguida por Ona, Tocomar, y por último Zapaleri.

En la Tabla 2 se puede observar la distribución por materia prima de las clases técnicas presentes en el conjunto. En ambos tipos de material la clase técnica predominante es la reducción bifacial, lo cual se debe en gran medida a la alta frecuencia de puntas de proyectil y preformas, luego sigue el trabajo no invasivo unifacial y el trabajo no invasivo bifacial. Finalmente, la reducción unifacial y el trabajo no invasivo alternante se observan solamente en artefactos de obsidiana. Es decir que la mayor diversidad de clases técnicas se observa en la materia prima no local.

Con respecto a las formas base de los instrumentos de obsidiana en los que fue posible observarlas $(\mathrm{N}=13)$, éstas consisten predominantemente en lascas angulares ( $n=11)$, seguidas por una lasca de arista y una lasca secundaria. En el caso de las 21 puntas de proyectil y preforma de obsidiana, dado que los lascados se superponen en el centro de las piezas en ambas caras, no es posible asegurar el tipo de forma base, pero según el tamaño de estos artefactos se podría suponer que las formas base fueron lascas. Lo mismo ocurre con las puntas de proyectil y preforma de materias primas locales. En los demás instrumentos de estos materiales, se pudo observar que también predominan las lascas angulares $(n=14)$, seguidas por una lasca de arista, una lasca secundaria, una lasca de dorso natural, y un núcleo pequeño. En consideración de los tamaños de los instrumentos, como fue mencionado, las puntas de proyectil tienen tamaño pequeño en su mayoría, mientras que los demás grupos tipológicos manifiestan tamaños pequeño y mediano pequeño, independientemente de la materia prima empleada, con excepción de dos artefactos de materia prima local que tienen un tamaño mediano pequeño. Finalmente, en relación a la presencia de reactivación, ésta sólo se registra en seis puntas de proyectil de obsidiana y dos de materiales locales.

\section{Discusión y conclusiones}

Como vimos, la diversidad de grupos tipológicos del conjunto no es alta, lo cual es esperable para ocupaciones logísticas, en las cuales las actividades tienden a ser acotadas, predecibles y programadas. Los instrumentos analizados, se pueden relacionar con prácticas extractivas y de procesamiento, algunos de los cuales (muescas, 
perforadores, raspadores) también pudieron ser utilizados para el tratamiento de artefactos de madera, como astiles $u$ otros componentes del sistema de armas. De hecho, la alta frecuencia de puntas de proyectil fracturadas (27) sugiere actividades de recambio de cabezales líticos en este sitio. En este marco, la obsidiana participa en la misma cantidad de grupos tipológicos que los materiales locales, y domina en seis de ellos. De esta manera, la obsidiana formó parte de casi toda la gama de instrumentos utilizados, a la par de las demás materias primas. En este conjunto, la variedad Quirón es la que predomina, lo cual es esperable teniendo en cuenta que su fuente es la más cercana al sitio. En este sentido resulta interesante el hecho de que la variedad que sigue en frecuencia es la de Ona, que se encuentra a una distancia superior a los $200 \mathrm{~km}$. De todos modos, se observa que todas las variedades participan en distintos grupos tipológicos.

En relación al grado de trabajo invertido en estos artefactos, se advierte que la obsidiana presenta mayor variabilidad de clases técnicas que las materias primas locales. No sólo fue utilizada para los artefactos de mayor inversión de energía como las puntas de proyectil, sino que la mitad de los artefactos de las demás clases técnicas en conjunto fue realizada con este material, e incluso los artefactos de menor formatización (trabajo no invasivo alternante) fueron hechos únicamente de obsidiana, en las variedades Ona y Zapaleri. En relación con esto, se observa que las formas base de los instrumentos con baja formatización es mayoritariamente la lasca angular, tanto en obsidiana como en los demás materiales. Este tipo de soporte no habría requerido de una formatización especial de los núcleos, de la misma manera que los filos de retoque marginal no requieren de una formatización de la forma base ni una imposición de forma específica para su confección. De esta manera, se puede afirmar que los instrumentos de baja formatización observados pertenecen a lo que se denomina diseño utilitario y corresponden a una estrategia tecnológica expeditiva (Escola, 2004b; Hocsman y Escola, 2006/2007).

Las puntas de proyectil y preformas son los artefactos formatizados predominantes (50\%), y representan casi la totalidad de la clase técnica de mayor inversión de energía, lo que coincide con lo observado en otros sitios de la región (Elías, 2010; Escola et al., 2006; Lavallé et al., 1997). Estos artefactos presentan muy baja variabilidad métrica y morfológica (Varde, 2017), por lo que se espera la presencia de requerimientos de extracción de la forma base orientados a producir soportes de dimensiones específicas y requerimientos de formatización de la forma base para facilitar la reducción bifacial de los filos. A su vez, la morfología de estas puntas de proyectil con partes diferenciadas (base escotada, aletas, limbo), y muy pautadas en virtud de su baja variabilidad, supone la presencia de requerimientos de imposición de forma. Además, son el único grupo tipológico que presenta reactivación de los filos, en algunos casos hasta el agotamiento. Por todo esto, se concluye que las puntas de proyectil de este conjunto son artefactos de diseño confiable, y corresponden a estrategias tecnológicas conservadas (Escola, 2004b; Hocsman y Escola, 2006/2007).

Como se observó en otro trabajo, los desechos de talla de obsidiana de este conjunto no superan la categoría microlasca, y la mayoría corresponde a lascas de reducción y formatización de filo por retoque (Varde et al, 2017). De esta manera, todas las lascas de obsidiana de tamaño superior a microlasca disponibles fueron utilizadas como instrumentos, tanto de diseño confiable como utilitario. Entonces, es posible proponer que hubo un aprovechamiento económico de la obsidiana por el uso de todas las piezas potencialmente utilizables mediante técnicas de formatización de alta y baja inversión de trabajo. Las materias primas locales, en cambio, no fueron usadas tan exhaustivamente, dado que presentan lascas de mayor tamaño sin signos claros de utilización, y núcleos no formales y sin agotamiento (Varde et al, 2017).

Una de las características de las estrategias expeditivas es la minimización de esfuerzo orientado a la selección y preparación de las materias primas (Escola, 2004b). En este trabajo se observa que en la ocupación tardía de Cueva Nacimiento 1, del total de instrumentos, la obsidiana alcanza o supera el 50\%, incluso cuando sólo se observan los artefactos de diseño utilitario. Además las distintas variedades de obsidiana participan en varios grupos tipológicos, sin exclusividad de una variedad para un tipo de clase técnica o grupo tipológico. Todos estos datos se destacan en comparación con varios trabajos realizados en otros sitios de la región, en los cuales la tendencia es a un predominio de materiales locales e inmediatos en los instrumentos expeditivos. El hecho de que la obsidiana haya sido utilizada en frecuencias tan altas, no sólo en los artefactos conservados sino también en aquellos de carácter expeditivo, indica que este material estuvo disponible y fue seleccionado en virtud de esa disponibilidad por sobre las materias primas locales de buena calidad (Andrefsky, 1994). Esto pudo ser posible únicamente como consecuencia de un acceso frecuente a estos materiales. Incluso es posible pensar que la baja frecuencia de reactivación en las puntas de proyectil pudo estar relacionado con esto, y que el mantenimiento de estos artefactos estuviera influenciado por la disponibilidad de materias primas de excelente calidad.

Un acceso frecuente a distintas variedades de obsidiana, algunas de fuentes muy lejanas como Ona y Zapaleri, ya fue documentado en el registro arqueológico temprano o formativo de poblaciones pastoriles de Santa Rosa de Los Pastos Grandes en la Puna de Salta (Mercuri, 2011). No se puede establecer, por el momento, la naturaleza del aprovisionamiento con una muestra limitada como la 
nuestra. Pero sí se puede proponer que, dada la distancia a las fuentes de obsidiana aquí analizadas, debió haberse tratado de algún mecanismo de intercambio. Diferentes modalidades de intercambio pudieron estar en juego entre las poblaciones pastoriles desde tiempos arcaicos tardíos, sea por caravaneo, por tráfico down-the-line, o por tráfico incorporado (Nielsen, 2013; Yacobaccio, 2012). En estos contextos, se ha planteado que el intercambio no sólo habría proporcionado bienes de subsistencia necesarios y complementarios para las poblaciones pastoriles de las tierras altas, sino también habría contribuido a formar y legitimar relaciones sociales, así como reforzar la reproducción social (Carbonelli, 2014; Nielsen, 2013). Por esto proponemos que los ocupantes de Cueva Nacimiento 1 pudieron estar involucrados de alguna manera en redes de interacción que permitieron el acceso a rocas provenientes de zonas lejanas, probablemente en paralelo a otros bienes de valor económico o simbólico. Para explorar esta hipótesis actualmente se está realizando el análisis de los restos botánicos y cerámicos.

Buenos Aires, 29 de marzo 2019.

\section{Agradecimientos}

Al Dr. Hernán Muscio, al Instituto de Arqueología de la FFyL de la UBA, y al CONICET, por hacer posible este trabajo, y a los evaluadores anónimos, cuyos comentarios lo enriquecieron. No obstante, todo lo expuesto aquí es de mi exclusiva autoría.

\section{Referencias citadas}

Andrefsky, W. (1994). Raw-material availability and the organization of technology. American Antiquity, 59 (1), 21-34.

Aschero, C. (1983). Ensayo para una clasificación morfológica de artefactos líticos. Apéndices $A$ y $B$. Cátedra de Ergología y Tecnología. Facultad de Filosofía y Letras, Universidad de Buenos Aires. Ms.

Aschero, C. y Hocsman, S. (2004). Revisando cuestiones tipológicas en torno a la clasificación de artefactos bifaciales. En Temas de arqueología. Análisis lítico, A. Acosta, D. Loponte y M. Ramos (Eds.), pp 7-25. Luján, Argentina: Universidad Nacional de Luján.

Carbonelli, P. (2014). Obsidianas y puntas de proyectil: sustancia y forma de las relaciones sociales en Las Pailas, Catamarca, Argentina. Revista Colombiana de Antropología 50: 117-137.

Elias, A. (2010). Estrategia tecnológicas y variabilidad de los conjuntos líticos de las sociedades tardías en Antofagasta de la Sierra (Provincia de Catamarca, Puna Meridional argentina). Tesis inédita de doctorado, Facultad de Filosofía y Letras, UBA.

Elias, A y Escola, P. (2010). Viejos y nuevos horizontes: obsidianas entre las sociedades agrícolas-pastoriles del Período Tardío en Antofagasta de la Sierra (provincia de Catamarca, Puna Meridional Argentina). Revista Española de Antropología Americana 40(2), 9-29.

Escola, P. (2004a). Variabilidad en la explotación y distribución de obsidianas en la Puna Meridional argentina. Estudios Atacameños 28, 9-24.

Escola, P. (2004b). La expeditividad y el registro arqueológico. Chungara 36, 49-60.

Escola, P., Elías, A. y Cohen, L. (2016). Obsidianas en el TardíoInka de Antofagasta de la Sierra (Puna meridional argentina): ¿fondo de cuenca versus sectores intermedios? Arqueología 22(1), 211-222

Escola, P, Elías, A. y Paulides, L. (2006). Bajo del Coypar II: Tendencias tecnológicas para el Tardío de Antofagasta de la Sierra (Catamarca). Werken 8: 5-24.

Escola, P., Glascock, M., Korstanje, A. y Sentinelli, N. (2009). Laguna Cavi y El Médano: obsidianas en circulación caravanera. En Arqueometría Latinoamericana: Segundo Congreso y Primero Latinoamericano, O. Palacios, C. Vasquez, T. Palacios, E. Cabanillas (Eds.), pp.103-108. Buenos Aires, Argentina: Comision Nacional de Energia Atomica.

Hocsman, S. y Escola, P. (2006- 2007). Inversión de trabajo y diseño en contextos líticos agro-pastoriles (Antofagasta de La Sierra, Catamarca). Cuadernos 21, 75- 91.

Lavallé, Daniele, Julien Mhichele, Claudine Karlin, Lidia C. García, Denise Pozzi-Escot y Michel Fontugne (1997). Entre desierto y quebrada. Primeros resultados de las excavaciones realizadas en el abrigo de Tomayoc (Puna de Jujuy, Argentina). Avances en Arqueología, 3: 9- 39.

López, G. (2008). Arqueología de cazadores y pastores en tierras altas: ocupaciones humanas a lo largo del Holoceno en Pastos Grandes, Puna de Salta, Argentina. BAR International Series 1854. Oxford: Oxbow books.

Mercuri, C. (2011). Diferencias y similitudes e las obsidianas del valle de SAC y la cuenca de Santa Rosa de los Pastos Grandes: aproximación a las redes de interacción social. En Arqueología de la Puna Argentina: perspectivas actuales en el estudio de la diversidad y el cambio cultural, G. López y H. Muscio (Eds.). BAR South American Archaeology Series 16, pp. 165-181. Oxford: Oxbow books.

Nielsen, A. (2013). Circulating Objects and the Constitution of South Andean Society (500 BC-AD 1550). En Merchants, markets and exchange in the pre-columbian world, K. G. Hirth y J. Pillsbury (Eds.), pp. 389-472. Dumbarton Oaks, Washington DC

Varde, M. (2017). Variación métrica en puntas de proyectil del período Tardío de la Puna de Salta y Jujuy: contribución teórico-metodológica para el estudio de transmisión cultural. Tesis inédita de licenciatura. Facultad de Filosofía y Letras, Universidad de Buenos Aires.

Varde, M., Seguí, S., y Rucci, L. (2017). Análisis del material arqueológico de la Cueva Nacimiento 1, Puna de Salta (Argentina), Período Tardío. Cuadernos del Instituto Nacional de Antropología y Pensamiento Latinoamericano - Series Especiales 4 (3), 31-41.

Yacobaccio, H. (2012). Intercambio y caravanas de llamas en el sur andino (3000-1000 AP). Chungara 16, 31-51.

Yacobaccio, H., P. Escola, F. Pereyra, M. Lazzari y M. Glascock (2004). Quest for ancient routes: obsidian sourcing research in Northwestern Argentina. Journal of Archaeological Science 31, 193-204. 